\title{
INTEGRABILITY OF ANTI-SELF-DUAL VACUUM EINSTEIN EQUATIONS WITH NONZERO COSMOLOGICAL CONSTANT: AN INFINITE HIERARCHY OF NONLOCAL CONSERVATION LAWS
}

\author{
I. KRASIL'SHCHIK AND A. SERGYEYEV
}

\begin{abstract}
We present an infinite hierarchy of nonlocal conservation laws for the Przanowski equation, an integrable second-order PDE locally equivalent to anti-self-dual vacuum Einstein equations with nonzero cosmological constant. The hierarchy in question is constructed using a nonisospectral Lax pair for the equation under study. As a byproduct, we obtain an infinite-dimensional differential covering over the Przanowski equation.
\end{abstract}

\section{INTRODUCTION}

Integrable systems play an important role in modern theoretical and mathematical physics, see e.g. 1, 4, 11, 13, 14, 20, 28, 31, 32, 35, 36, 37, and this is particularly true for integrable systems in four independent variables a.k.a. $3+1$ dimensions, cf. e.g. [11, 25, 32, 36] and references therein, since according to general relativity our spacetime is four-dimensional. Moreover, a number of integrable (3+1)-dimensional systems of immediate relevance for physics arises within general relativity upon imposition of (anti)self-duality conditions, see, for instance, [1, 3, 25, and references therein, as is the case for the Przanowski equation studied below.

Namely, Przanowski [30 has shown that locally every anti-self-dual Einstein four-manifold $(M, g)$ admits a compatible complex structure and the metric has the form

$$
g=2\left(u_{w \bar{w}} d w d \bar{w}+u_{w \bar{z}} d w d \bar{z}+u_{z \bar{w}} d z d \bar{w}+\left(u_{z \bar{z}}+\frac{2}{\Lambda} \exp (\Lambda u)\right) d z d \bar{z}\right) .
$$

Here $u=u(w, \bar{w}, z, \bar{z})$ is a real function on $M, w$ and $z$ are holomorphic coordinates on $M$ and $\bar{w}$ and $\bar{z}$ denote their complex conjugates; $\Lambda \neq 0$ is the cosmological constant, cf. e.g. [8] and references therein. As usual, the subscripts indicate partial derivatives, e.g. $u_{w}=\frac{\partial u}{\partial w}$ etc.

The metric (11) is, see [30], an anti-self-dual Einstein metric if and only if $u$ satisfies the Przanowski equation

$$
u_{z \bar{w}} u_{w \bar{z}}-u_{w \bar{w}}\left(u_{z \bar{z}}+\frac{2}{\Lambda} \exp (\Lambda u)\right)+u_{w} u_{\bar{w}} \exp (\Lambda u)=0,
$$

which is a subject of intense research, see e.g. [2, 12, 21] and references therein.

We leave aside the case of $\Lambda=0$ as then, upon having imposed (anti)self-duality on the metric one has to use a normal form of the metric different from (11) and arrives instead of (2) at a different PDE, cf. e.g. [1, 21, 25, 29, 33, 34] and references therein.

Hoegner [12] has established integrability of (2) by constructing a nonisospectral Lax pair for (2) of the form

$$
l_{i} \psi=0, \quad i=1,2,
$$

where $\psi=\psi(z, w, \bar{z}, \bar{w}, \xi), Q=-u_{w} u_{\bar{w}} \exp (\Lambda u)$, and

$$
\begin{aligned}
& l_{1}=\partial_{w}-\xi \frac{u_{w \bar{z}}}{Q} \partial_{\bar{w}}+\xi \frac{u_{w \bar{w}}}{Q} \partial_{\bar{z}}+\left(\frac{\partial_{w} Q+\exp (\Lambda u) u_{w} u_{w \bar{w}}}{Q}-\frac{u_{w \bar{w}}}{u_{\bar{w}}}\right) \xi \partial_{\xi}, \\
& l_{2}=\partial_{z}-\frac{\xi}{Q}\left(u_{z \bar{z}}+\frac{2}{\Lambda} \exp (\Lambda u)\right) \partial_{\bar{w}}+\xi \frac{u_{z \bar{w}}}{Q} \partial_{\bar{z}}+\left(\frac{\partial_{z} Q+\exp (\Lambda u)\left(u_{w} u_{z \bar{w}}-u_{\bar{w}} \xi\right)}{Q}-\frac{u_{z \bar{w}}}{u_{\bar{w}}}+\frac{\xi}{u_{w}}\right) \xi \partial_{\xi} .
\end{aligned}
$$

Here $\xi$ is an additional independent variable which plays the role of (variable) spectral parameter, see [9, 24, 32, 37] and references therein; we stress that $u_{\xi} \equiv 0$.

The presence of the above Lax pair makes it possible, at least in principle, to obtain exact solutions for (2) using the inverse scattering transform, cf. e.g. 24] and references therein, or the twistorial methods, see, for example, [3, 11, 12, 25] and references therein.

Date: June 3, 2019. 
Existence of infinite hierarchies of conservation laws is a well-known feature of integrable systems, cf. e.g. [20, 28] and references therein, and we show below how to construct such a hierarchy for the Przanowski equation using a modification of the above Lax pair. Moreover, we prove that the conservation laws in question are nontrivial and linearly independent.

The rest of the article is organized as follows. In Section 1 we construct an infinite hierarchy of the nonlocal conservation laws in question. In Section 2 we set the stage for Section 3 and our main result, Theorem 1, establishing linear independence and nontriviality for the conservation laws from the hierarchy in question. Section 4 contains discussion.

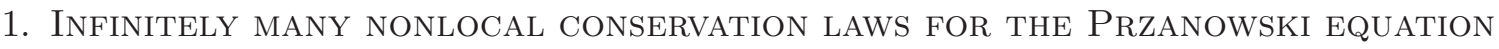

Proposition 1. Equation (2) admits a Lax pair $L_{j} \chi=0, j=1,2$, where $\chi=\chi(z, \bar{z}, w, \bar{w}, p)$ and

$$
\begin{aligned}
L_{1}= & \partial_{w}-\frac{p u_{w \bar{w}}}{u_{\bar{w}}^{2}} \partial_{\bar{z}}+\frac{p u_{w \bar{z}}}{u_{\bar{w}}^{2}} \partial_{\bar{w}}+\frac{\left(u_{\bar{w} \bar{w}} u_{w \bar{z}}-u_{w \bar{w}} u_{\bar{w} \bar{z}}+\Lambda u_{\bar{w}}\left(u_{\bar{z}} u_{w \bar{w}}-u_{\bar{w}} u_{w \bar{z}}\right)\right) p^{2}}{u_{\bar{w}}^{3}} \partial_{p} \\
L_{2}= & \partial_{z}-\frac{p u_{\bar{w} z}}{u_{\bar{w}}^{2}} \partial_{\bar{z}}+\frac{\left(u_{\bar{w} z} u_{w \bar{z}}+\exp (\Lambda u) u_{w} u_{\bar{w}}\right) p}{u_{\bar{w}}^{2} u_{w \bar{w}}} \partial_{\bar{w}} \\
& -\frac{\left(u_{\bar{w} z}\left(u_{w \bar{w}} u_{\bar{w} \bar{z}}-u_{\bar{w} \bar{w}} u_{w \bar{z}}-\Lambda u_{\bar{w}}\left(u_{\bar{z}} u_{w \bar{w}}-u_{\bar{w}} u_{w \bar{z}}\right)\right)-\exp (\Lambda u) u_{\bar{w}}\left(u_{\bar{w}} u_{w \bar{w}}+u_{w} u_{\bar{w} \bar{w}}-\Lambda u_{w} u_{\bar{w}}^{2}\right)\right) p^{2}}{u_{\bar{w}}^{3} u_{w \bar{w}}} \partial_{p} .
\end{aligned}
$$

Proof. It suffices to observe that $L_{i}$ are related to $l_{i}$ by the change of variables $p=\xi \exp (\Lambda u) u_{w} / u_{\bar{w}}$.

The Lax operators $L_{i}$ enjoy a simpler structure than $l_{i}$. Indeed, they can be written as

$$
\begin{aligned}
L_{1} & =\partial_{w}+p\left(\frac{1}{u_{\bar{w}}}\right)_{w} \partial_{\bar{z}}-p\left(\omega_{1}\right)_{w} \partial_{\bar{w}}+p^{2}\left(\frac{u_{\bar{w}}\left(2 \Lambda u_{\bar{z}}+\left(\omega_{1}\right)_{\bar{w}}\right)-u_{\bar{w} \bar{z}}}{2 u_{\bar{w}}^{2}}\right)_{w} \partial_{p}, \\
L_{2} & =\partial_{z}+p\left(\frac{1}{u_{\bar{w}}}\right)_{z} \partial_{\bar{z}}-p\left(\omega_{1}\right)_{z} \partial_{\bar{w}}+p^{2}\left(\frac{u_{\bar{w}}\left(2 \Lambda u_{\bar{z}}+\left(\omega_{1}\right)_{\bar{w}}\right)-u_{\bar{w} \bar{z}}}{2 u_{\bar{w}}^{2}}\right)_{z} \partial_{p},
\end{aligned}
$$

where $\omega_{1}$ is defined by the formulas

$$
\begin{aligned}
\left(\omega_{1}\right)_{w} & =\frac{u_{w \bar{z}}}{u_{\bar{w}}^{2}}, \\
\left(\omega_{1}\right)_{z} & =\frac{\left(u_{\bar{w} z} u_{w \bar{z}}+\exp (\Lambda u) u_{w} u_{\bar{w}}\right)}{u_{\bar{w}}^{2} u_{w \bar{w}}},
\end{aligned}
$$

i.e., the quantity $\omega_{1}$ is a nonlocal variable, namely, a potential for the following local conservation law for (2):

$$
\left(\frac{u_{w \bar{z}}}{u_{\bar{w}}^{2}}\right)_{z}=\left(\frac{\left(u_{\bar{w} z} u_{w \bar{z}}+\exp (\Lambda u) u_{w} u_{\bar{w}}\right)}{u_{\bar{w}}^{2} u_{w \bar{w}}}\right)_{w} .
$$

We stress that the derivatives $\left(\omega_{1}\right)_{\bar{w}}$ and $\left(\omega_{1}\right)_{\bar{z}}$ are not determined by (4), and hence all quantities $\left(\omega_{1}\right)_{\bar{w}^{k} \bar{z}^{l}}$ for $k, l=0,1,2, \ldots$ are functionally independent nonlocal variables. In a similar fashion we have infinitely many nonlocal variables $\chi_{\bar{w}^{k} \bar{z}^{l}}^{s}$ in (11) below. This is a fairly common phenomenon for nonlocal variables associated with partial differential systems in more than two independent variables, cf. e.g. Remark 3.9 in [20].

Substituting a formal Taylor expansion $\chi=\sum_{i=0}^{\infty} \chi_{i} p^{i}$ into the equations $L_{j} \chi=0$ shows that $\chi_{0}=\chi_{0}^{0}(\bar{w}, \bar{z})$ is an arbitrary smooth function of $\bar{w}$ and $\bar{z}$, and $\chi_{1}$ satisfies the equations

$$
\left(\chi_{1}\right)_{w}=\frac{u_{w \bar{w}}}{u_{\bar{w}}^{2}}\left(\chi_{0}^{0}\right)_{\bar{z}}-\frac{u_{w \bar{z}}}{u_{\bar{w}}^{2}}\left(\chi_{0}^{0}\right)_{\bar{w}}
$$

$$
\left(\chi_{1}\right)_{z}=\frac{u_{\bar{w} z}}{u_{\bar{w}}^{2}}\left(\chi_{0}^{0}\right)_{\bar{z}}-\frac{\left(u_{\bar{w} z} u_{w \bar{z}}+\exp (\Lambda u) u_{w} u_{\bar{w}}\right)}{u_{\bar{w}}^{2} u_{w \bar{w}}}\left(\chi_{0}^{0}\right)_{\bar{w}}
$$

whence

$$
\chi_{1}=-\frac{\left(\chi_{0}^{0}\right)_{\bar{z}}}{u_{\bar{w}}}-\omega_{1}\left(\chi_{0}^{0}\right)_{\bar{w}}+\chi_{1}^{0},
$$

where $\chi_{1}^{0}(\bar{w}, \bar{z})$ is again an arbitrary smooth function of $\bar{w}$ and $\bar{z}$. 
We now see that $\chi_{i}$ for $i=2,3, \ldots$ satisfy the recursion relations

$$
\begin{aligned}
\left(\chi_{i}\right)_{w} & =-\left(\frac{1}{u_{\bar{w}}}\right)_{w}\left(\chi_{i-1}\right)_{\bar{z}}+\left(\omega_{1}\right)_{w}\left(\chi_{i-1}\right)_{\bar{w}}-\left(\frac{u_{\bar{w}}\left(2 \Lambda u_{\bar{z}}+\left(\omega_{1}\right)_{\bar{w}}\right)-u_{\bar{w} \bar{z}}}{2 u_{\bar{w}}^{2}}\right)_{w}(i-1) \chi_{i-1}, \\
\left(\chi_{i}\right)_{z} & =-\left(\frac{1}{u_{\bar{w}}}\right)_{z}\left(\chi_{i-1}\right)_{\bar{z}}+\left(\omega_{1}\right)_{z}\left(\chi_{i-1}\right)_{\bar{w}}-\left(\frac{u_{\bar{w}}\left(2 \Lambda u_{\bar{z}}+\left(\omega_{1}\right)_{\bar{w}}\right)-u_{\bar{w} \bar{z}}}{2 u_{\bar{w}}^{2}}\right)_{z}(i-1) \chi_{i-1}
\end{aligned}
$$

Thus, we have an infinite-dimensional (differential) covering over (2) defined by (7), cf. e.g. [7, 20] for general background on coverings.

System (7) gives rise to an infinite hierarchy of nonlocal conservation laws for (21):

$$
\left(A_{k, r, s}\right)_{z}=\left(B_{k, r, s}\right)_{w}
$$

where $k=1,2,3, \ldots, r, s=0,1,2,3, \ldots$, and $A_{k, r, s}$ and $B_{k, r, s}$ are defined by the following relations:

$$
A_{k, r+1, s}=\left(A_{k, r, s}\right)_{\bar{z}}, \quad A_{k, r, s+1}=\left(A_{k, r, s}\right)_{\bar{w}}, \quad B_{k, r+1, s}=\left(B_{k, r, s}\right)_{\bar{z}}, \quad B_{k, r, s+1}=\left(B_{k, r, s}\right)_{\bar{w}}, \quad k, r, s=1,2,3, \ldots,
$$

and for $k=1,2,3, \ldots$ we set

$$
\begin{aligned}
& A_{k, 0,0}=-\left(\frac{1}{u_{\bar{w}}}\right)_{w}\left(\chi_{k}\right)_{\bar{z}}+\left(\omega_{1}\right)_{w}\left(\chi_{k}\right)_{\bar{w}}-\left(\frac{u_{\bar{w}}\left(2 \Lambda u_{\bar{z}}+\left(\omega_{1}\right)_{\bar{w}}\right)-u_{\bar{w} \bar{z}}}{2 u_{\bar{w}}^{2}}\right)_{w} k \chi_{k}, \\
& B_{k, 0,0}=-\left(\frac{1}{u_{\bar{w}}}\right)_{z}\left(\chi_{k}\right)_{\bar{z}}+\left(\omega_{1}\right)_{z}\left(\chi_{k}\right)_{\bar{w}}-\left(\frac{u_{\bar{w}}\left(2 \Lambda u_{\bar{z}}+\left(\omega_{1}\right)_{\bar{w}}\right)-u_{\bar{w} \bar{z}}}{2 u_{\bar{w}}^{2}}\right)_{z} k \chi_{k} .
\end{aligned}
$$

The conservation laws (8) are linearly independent and nontrivial, as we are going to establish in Theorem 1 below.

In closing note that (2) enjoys an obvious discrete symmetry $w \leftrightarrow \bar{w}, z \leftrightarrow \bar{z}$ which however does not extend to its Lax operators $l_{i}$ or $L_{i}$. This implies that there exists another infinite hierarchy of nonlocal conservation laws for (2) obtained from (8) by the simultaneous swap $w \leftrightarrow \bar{w}, z \leftrightarrow \bar{z}$.

\section{Nontriviality OF CONSERVATION LAWS: PRELIMINARIES}

2.1. Simplifications. Before proceeding further, notice that the problem under study admits some useful simplifications. Namely, note that

(1) Under the rescaling $u \mapsto \Lambda u$ equation (2) transforms into

$$
u_{z \bar{w}} u_{w \bar{z}}-u_{w \bar{w}}\left(u_{z \bar{z}}+2 e^{u}\right)+u_{w} u_{\bar{w}} e^{u}=0
$$

and thus we can set $\Lambda=1$ in all subsequent computations without loss of generality.

(2) Coverings (6) and (4) are equivalent in the sense of [19], which inter alia implies that we can set without loss of generality $\chi_{0}^{0}=-\bar{w}, \chi_{1}^{0}=0$.

Thus, the infinite-dimensional covering defined by (66) and (7) boils down to

$$
\chi_{1, w}=\frac{u_{w \bar{z}}}{u_{\bar{w}}^{2}}, \quad \chi_{1, z}=\frac{\left(u_{\bar{w} z} u_{w \bar{z}}+u_{w} u_{\bar{w}} e^{u}\right)}{u_{\bar{w}}^{2} u_{w \bar{w}}}
$$

and

$$
\begin{aligned}
\chi_{i, w} & =-\left(\frac{1}{u_{\bar{w}}}\right)_{w}\left(\chi_{i-1}\right)_{\bar{z}}+\left(\chi_{1}\right)_{w}\left(\chi_{i-1}\right)_{\bar{w}}-\left(\frac{u_{\bar{w}}\left(2 u_{\bar{z}}+\left(\chi_{1}\right)_{\bar{w}}\right)-u_{\bar{w} \bar{z}}}{2 u_{\bar{w}}^{2}}\right)_{w}(i-1) \chi_{i-1}, \\
\chi_{i, z} & =-\left(\frac{1}{u_{\bar{w}}}\right)_{z}\left(\chi_{i-1}\right)_{\bar{z}}+\left(\chi_{1}\right)_{z}\left(\chi_{i-1}\right)_{\bar{w}}-\left(\frac{u_{\bar{w}}\left(2 u_{\bar{z}}+\left(\chi_{1}\right)_{\bar{w}}\right)-u_{\bar{w} \bar{z}}}{2 u_{\bar{w}}^{2}}\right)_{z}(i-1) \chi_{i-1}
\end{aligned}
$$

for $i>1$. 
2.2. Coordinates and total derivatives. We rewrite (2), where we set $\Lambda=1$ as per Section 2.1, in the form

$$
u_{z \bar{z}}=\frac{u_{w \bar{z}} u_{z \bar{w}}-\left(u_{w} u_{\bar{w}}-2 u_{w \bar{w}}\right) e^{u}}{u_{w \bar{w}}}
$$

and choose internal coordinates on the associated diffiety $\mathscr{E}$, i.e., the infinite prolongation of (9)), as follows (see e.g. [7] for the background on geometry of diffieties):

$$
u_{w^{i} \bar{w}^{j}}=\frac{\partial^{i+j} u}{\partial w^{i} \partial \bar{w}^{j}}, \quad u_{w^{i} \bar{w}^{j} z^{k}}=\frac{\partial^{i+j+1} u}{\partial w^{i} \partial \bar{w}^{j} \partial z^{k}}, \quad u_{w^{i} \bar{w}^{j} \bar{z}^{k}}=\frac{\partial^{i+j+k} u}{\partial w^{i} \partial \bar{w}^{j} \partial \bar{z}^{k}},
$$

where $i, j \geq 0, k>0$.

Then the total derivatives on $\mathscr{E}$ read

$$
\begin{aligned}
& D_{w}=\frac{\partial}{\partial w}+\sum_{i, j, k}\left(u_{w^{i+1} \bar{w}^{j}} \frac{\partial}{\partial u_{w^{i} \bar{w}^{j}}}+u_{w^{i+1} \bar{w}^{j} z^{k}} \frac{\partial}{\partial u_{w^{i} \bar{w}^{j} z^{k}}}+u_{w^{i+1} \bar{w}^{j} \bar{z}^{k}} \frac{\partial}{\partial u_{w^{i} \bar{w}^{j} \bar{z}^{k}}}\right), \\
& D_{\bar{w}}=\frac{\partial}{\partial \bar{w}}+\sum_{i, j, k}\left(u_{w^{i} \bar{w}^{j+1}} \frac{\partial}{\partial u_{w^{i} \bar{w}^{j}}}+u_{w^{i} \bar{w}^{j+1} z^{k}} \frac{\partial}{\partial u_{w^{i} \bar{w}^{j} z^{k}}}+u_{w^{i} \bar{w}^{j+1} \bar{z}^{k}} \frac{\partial}{\partial u_{w^{i} \bar{w}^{i} \bar{z}^{k}}}\right), \\
& D_{z}=\frac{\partial}{\partial z}+\sum_{i, j, k}\left(u_{w^{i} \bar{w}^{j} z^{1}} \frac{\partial}{\partial u_{w^{i} \bar{w}^{j}}}+u_{w^{i} \bar{w}^{j} z^{k+1}} \frac{\partial}{\partial u_{w^{i} \bar{w}^{j} z^{k}}}+D_{w}^{i} D_{\bar{w}^{j}} D_{\bar{z}}^{k-1}(R) \frac{\partial}{\partial u_{w^{i} \bar{w}^{j} \bar{z}^{k}}}\right), \\
& D_{\bar{z}}=\frac{\partial}{\partial \bar{z}}+\sum_{i, j, k}\left(u_{w^{i} \bar{w}^{j} \bar{z}^{1}} \frac{\partial}{\partial u_{w^{i} \bar{w}^{j}}}+D_{w}^{i} D_{\bar{w}}^{j} D_{z}^{k-1}(R) \frac{\partial}{\partial u_{w^{i} \bar{w}^{j} z^{k}}}+u_{w^{i} \bar{w}^{j} \bar{z}^{k+1}} \frac{\partial}{\partial u_{w^{i} \bar{w}^{j} \bar{z}^{k}}}\right),
\end{aligned}
$$

where

$$
R=\frac{u_{w \bar{z}} u_{z \bar{w}}-\left(u_{w} u_{\bar{w}}-2 u_{w \bar{w}}\right) e^{u}}{u_{w \bar{w}}}
$$

is the right-hand side of (9).

To introduce nonlocal variables, we, for convenience of notation, do some relabeling, namely, let $\chi^{1}=\omega_{1}$ and $\chi^{s}=\chi_{s-1}, s \geq 2$. Then the nonlocal variables employed below are

$$
\chi_{\bar{w}^{k} \bar{z}^{l}}^{s}=\frac{\partial^{k+l} \chi^{s}}{\partial \bar{w}^{k} \partial \bar{z}^{l}}, \quad i \geq 1, \quad k, l \geq 0 .
$$

The total derivatives lifted to the covering equation are

$$
\tilde{D}_{w}=D_{w}+W, \quad \tilde{D}_{\bar{w}}=D_{\bar{w}}+\bar{W}, \quad \tilde{D}_{z}=D_{z}+Z, \quad \tilde{D}_{\bar{z}}=D_{\bar{z}}+\bar{Z},
$$

where the nonlocal tails

$$
W=\sum W_{s}^{k, l} \frac{\partial}{\partial \chi_{\bar{w}^{k} \bar{z}^{l}}^{s}}, \quad \bar{W}=\sum \bar{W}_{s}^{k, l} \frac{\partial}{\partial \chi_{\bar{w}^{k} \bar{z}^{l}}^{s}}, \quad Z=\sum Z_{s}^{k, l} \frac{\partial}{\partial \chi_{\bar{w}^{k} \bar{z}^{l}}^{s}}, \quad \bar{Z}=\sum \bar{Z}_{s}^{k, l} \frac{\partial}{\partial \chi_{\bar{w}^{k} \bar{z}^{l}}^{s}}
$$

are defined by the formulas

$$
W_{s}^{k, l}=\tilde{D}_{\bar{w}}^{k} \tilde{D}_{\bar{z}}^{l}\left(\chi_{w}^{s}\right), \quad \tilde{W}_{s}^{k, l}=\chi_{\bar{w}^{k+1} \bar{z}^{l}}^{s}, \quad Z_{s}^{k, l}=\tilde{D}_{\bar{w}}^{k} \tilde{D}_{\bar{z}}^{l}\left(\chi_{z}^{s}\right), \quad \bar{Z}_{s}^{k, l}=\chi_{\bar{w}^{k} \bar{z}^{l+1}}^{s}
$$

and $\chi_{w}^{s}, \chi_{z}^{s}$ are as defined above and take the form

$$
\begin{aligned}
\chi_{w}^{1} & =\frac{u_{w \bar{z}}}{u_{\bar{w}}^{2}}, \\
\chi_{z}^{1} & =\frac{u_{\bar{w} z} u_{w \bar{z}}+u_{w} u_{\bar{w}} e^{u}}{u_{\bar{w}}^{2} u_{w^{1} \bar{w}^{1}}} \\
\chi_{w}^{s} & =-A_{w} \chi_{\bar{z}}^{\bar{s}-1}+\chi_{w}^{1} \chi_{\bar{w}}^{s-1}-(s-1) B_{w} \chi^{s-1}, \\
\chi_{z}^{s} & =-A_{z} \chi_{\bar{z}}^{s-1}+\chi_{z}^{1} \chi_{\bar{w}}^{-1}-(s-1) B_{z} \chi^{s-1}
\end{aligned}
$$

here $s \geq 2$ and

$$
A=\frac{1}{u_{\bar{w}}}, \quad B=\frac{u_{\bar{w}}\left(2 u_{\bar{z}}+\chi_{\bar{w}}^{1}\right)-u_{\bar{w} \bar{z}}}{2 u_{\bar{w}}^{2}}
$$

while

$$
\left(\chi_{\bar{w}^{k} \bar{z}^{l}}^{s}\right)_{w}=\tilde{D}_{\bar{w}}^{k} \tilde{D}_{\bar{z}}^{l}\left(R W^{s}\right), \quad\left(\chi_{\bar{w}^{k} \bar{z}^{l}}^{s}\right)_{z}=\tilde{D}_{\bar{w}}^{k} \tilde{D}_{\bar{z}}^{l}\left(R Z^{s}\right),
$$

where $R W^{s}, R Z^{s}$ are the right-hand sides of (10). 
In what follows we shall need the following presentation of the coefficients $R W^{s}$ :

$$
\begin{aligned}
R W^{1} & =\frac{u_{w \bar{z}}}{u_{\bar{w}}^{2}} \\
R W^{2} & =\frac{1}{2} \cdot\left(\frac{1}{u_{\bar{w}}^{2}}-\frac{1}{u_{\bar{w}}^{3}}\right) u_{w \bar{w} \bar{z}} \chi^{1}+\left(\frac{u_{w \bar{z}}}{u_{\bar{w}}^{2}}+\frac{1}{2} \frac{u_{w \bar{w}}}{u_{\bar{w}}^{2}}\right) \chi_{\bar{w}}^{1}+\frac{u_{w \bar{w}}}{u_{\bar{w}}^{2}} \chi_{\bar{z}}^{1}+o, \\
R W^{s} & =\frac{1}{2} \cdot\left(\frac{1}{u_{\bar{w}}^{2}}-\frac{1}{u_{\bar{w}}^{3}}\right) u_{w \bar{w} \bar{z}}(s-1) \chi^{s-1}+\frac{u_{w \bar{z}}}{u_{\bar{w}}^{2}} \chi_{\bar{w}}^{s-1}+\frac{u_{w \bar{w}}}{u_{\bar{w}}^{2}} \chi_{\bar{z}}^{s-1}+o,
\end{aligned}
$$

where $s>2$ and $o$ denotes the terms of lower jet order both in $u$ and $\chi^{s}$ which are inessential for the subsequent computations.

\section{NONTRIVIALITY OF CONSERVATION LAWS: THE PROOF}

Equations (10)-(11) define an infinite family of (nonlocal) conservation laws

$$
\omega_{k, l}^{i}=\tilde{D}_{\bar{w}}^{k} \tilde{D}_{\bar{z}}^{l}\left(R W^{i}\right) d w+\tilde{D}_{\bar{w}}^{k} \tilde{D}_{\bar{z}}^{l}\left(R Z^{i}\right) d z, \quad i \geq 1, \quad k, l \geq 0
$$

for equation (9).

In other words, on $\mathscr{E}$ we have

$$
\tilde{D}_{z}\left(\rho_{k, l}^{i}\right)-\tilde{D}_{w}\left(\sigma_{k, l}^{i}\right)=0
$$

where $\rho_{k, l}^{i}=\tilde{D}_{\bar{w}}^{k} \tilde{D}_{\bar{z}}^{l}\left(R W^{i}\right)$ and $\sigma_{k, l}^{i}=\tilde{D}_{\bar{w}}^{k} \tilde{D}_{\bar{z}}^{l}\left(R Z^{i}\right)$.

Note that all these conservation laws are two-component in the sense that expressions (14) involve only two total derivatives, $\tilde{D}_{z}$ and $\tilde{D}_{w}$, out of four.

It could be of interest to find out whether (9) also has three- or four-component conservation laws, cf. e.g. [22, 23] and references therein, and to explore nonlocal symmetries for (99) involving nonlocal variables $\chi_{\bar{w}^{k} \bar{z}^{l}}^{s}$ being potentials for the conservation laws (13). Note that our computations show that there are no three- or four-component local conservation laws of order up to four for (9), and we strongly suspect that none exist even if we proceed to higher orders, cf. the discussion at the end of Section 4 . However, the matter of existence of nonlocal three- or four-component conservation laws for (9) remains an interesting open problem.

We now intend to prove that the system of the conservation laws under study is nontrivial. Let us clarify this claim.

The system of conservation laws $\omega_{k, l}^{1}$ defines the tower of coverings

$$
\mathscr{E}=\mathscr{E}^{0} \longleftarrow \mathscr{E}_{1} \longleftarrow \mathscr{E}_{2}^{1} \longleftarrow \ldots \longleftarrow \mathscr{E}_{i}^{1} \longleftarrow \ldots \longleftarrow \mathscr{E}^{1}
$$

where $\mathscr{E}$ is the infinite prolongation of the Przanowski equation, while the covering equations $\mathscr{E}_{i}^{1}$ contain the nonlocal variables $\chi_{\bar{w}^{k} \bar{z}^{l}}^{1}, 0 \leq k+l \leq i$, with $\mathscr{E}^{1}$ being the inverse limit. In a similar fashion, we define by induction the towers

$$
\mathscr{E}^{s} \longleftarrow \mathscr{E}_{1}^{s+1} \longleftarrow \mathscr{E}_{2}^{s+1} \longleftarrow \ldots \longleftarrow \mathscr{E}_{i}^{s+1} \longleftarrow \ldots \longleftarrow \mathscr{E}^{s+1}
$$

and

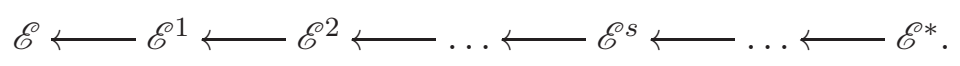

We are going to prove the following

Theorem 1. For any $i \geq 0$, an arbitrary finite system of conservation laws $\omega_{k, l}^{i+1}$ of the equation $\mathscr{E}^{i}$ is linearly independent.

The nontriviality of these conservation laws is, in view of their structure, see (8), a straightforward consequence of their linear independence.

The proof of Theorem 1 will be based on the following

Proposition 2 (see [16], cf. also [17, 18]). Let $\mathscr{E}$ be a differentially connected equation 1 . The conservation laws $\omega_{k, l}^{i}$ are mutually independent in the sense of Theorem 1 if and only if $\mathscr{E}^{*}$ is differentially connected as well, i.e., the

\footnotetext{
${ }^{1}$ Recall that an equation is called differentially connected if the only functions that are invariant with respect to all total derivatives on $\mathscr{E}$ are constants.
} 
only solutions of the system

$$
\tilde{D}_{w}(f)=\tilde{D}_{\bar{w}}(f)=\tilde{D}_{z}(f)=\tilde{D}_{\bar{z}}(f)=0, \quad f \in C^{\infty}\left(\mathscr{E}^{*}\right)
$$

are constants.

Proof of Theorem 1. We begin the proof with an obvious observation that the Przanowski equation (9) is differentially connected. Before proceeding further with the proof of the theorem, let us briefly describe the outline of the former. Namely, we will prove that the space $\operatorname{ker} \tilde{D}_{w}$ consists of functions that depend on $\bar{w}, z$, and $\bar{z}$ only, from where the desired result immediately follows. To this end, we will perform double induction: on $i$ in (16) and on $s$ in (15) for each $i$. The case of $i=1$ is special and is considered separately. So, the base of induction is $i=2$.

Let us employ the notation $\mathscr{F}\left(\mathscr{U}, n_{1}, \ldots, n_{s}\right)$ for the space of functions that belong to $\operatorname{ker} \tilde{D}_{w}$ and depend on a finite set $\mathscr{U}$ of internal coordinates in $\mathscr{E}$ and of nonlocal variables $\chi_{\bar{w}^{k} \bar{z}^{l}}^{\alpha}, \alpha=1, \ldots, s, k+l \leq n_{\alpha}$.

Lemma 1. If $F \in \mathscr{F}\left(\mathscr{U}, n_{1}, \ldots, n_{s}\right)$ then $n_{s}>n_{s-1}>\cdots>n_{1}$.

Proof of Lemma 1. The desired result is a straightforward consequence of the defining equations (10) and (11).

Let us now pass to the proof of the theorem.

Step $1(s=1)$. We prove here by induction on $n_{1}$ that the conservation laws $\omega_{k, l}^{1}$ are linearly independent. Denote $n_{1}=n$ and perform induction on $n$.

First, let $n=0$. Then

$$
\tilde{D}_{w}(F)=D_{w}(F)+\frac{u_{w \bar{z}}}{u_{\bar{w}}^{2}} \frac{\partial F}{\partial \chi^{1}}=0 .
$$

This means that the set $\mathscr{U}$ may consist of the variables $w, \bar{w}, z, \bar{z}$, and $u_{\bar{z}}$ only, i.e.,

$$
\frac{\partial F}{\partial w}+u_{w \bar{z}} \frac{\partial F}{\partial u_{\bar{z}}}+\frac{u_{w \bar{z}}}{u_{\bar{w}}^{2}} \frac{\partial F}{\partial \chi^{1}}=0
$$

and consequently

$$
\frac{\partial F}{\partial u_{\bar{z}}}+\frac{1}{u_{\bar{w}}^{2}} \frac{\partial F}{\partial \chi^{1}}=0
$$

Thus, $F=F(\bar{w}, z, \bar{z})$.

Next, let $n>0$. Then

$$
\tilde{D}_{w}=D_{w}+\sum_{k+l \leq n} \tilde{D}_{\bar{w}}^{k} \tilde{D}_{\bar{w}}^{l}\left(\frac{u_{w \bar{z}}}{u_{\bar{w}}^{2}}\right) \frac{\partial}{\partial \chi_{\bar{w}^{k} \bar{z}^{l}}^{1}} .
$$

In this expession, the variables $u_{\sigma}$ of the maximal jet order are $u_{w \bar{w}^{k} \bar{z}^{l+1}}, k+l=n$. Consequently, $F$ is invariant with respect to the vector fields

$$
Z_{k, l}=\frac{\partial}{\partial u_{\bar{w}^{k} \bar{z}^{l+1}}}+\frac{1}{u_{\bar{w}}^{2}} \cdot \frac{\partial}{\partial \chi_{\bar{w}^{k} \bar{z}^{l}}^{1}}, \quad k+l=n .
$$

Now notice that the coefficients of $\tilde{D}_{w}$ at $\partial / \partial \chi_{\bar{w}^{k} \bar{z}^{l}}^{1}$ are independent of the variables $u_{w \bar{w}^{i}}, i>0$. From this fact it immediately follows that $F$ cannot depend on $u_{\bar{w}}^{i}, i>0$. Hence, taking commutators of the vector fields $Z_{k, l}$ with $\partial / \partial u_{\bar{w}}$ we obtain that $F$ is invariant with respect to the derivations $\partial / \partial \chi_{\bar{w}^{k} \bar{z}^{l}}^{1}$, and this completes the induction step.

Step $2(s=2$, the base of induction on $s)$. Let now $F \in \mathscr{F}\left(\mathscr{U}, n_{1}, n_{2}\right)$.

Lemma 2. $n_{1}=n_{2}+1$.

Proof of Lemma 国. Consider the total derivative

$$
\tilde{D}_{w}(F)=\frac{\partial F}{\partial w}+\sum_{\mathscr{U}} u_{w \sigma} \frac{\partial F}{\partial u_{\sigma}}+\sum_{k+l \leq n_{1}} \tilde{D}_{\bar{w}}^{k} \tilde{D}_{\bar{z}}^{l}\left(R W^{1}\right) \frac{\partial F}{\partial \chi_{\bar{w}^{k} \bar{z}^{l}}^{1}}+\sum_{k+l \leq n_{2}} \tilde{D}_{\bar{w}}^{k} \tilde{D}_{\bar{z}}^{l}\left(R W^{2}\right) \frac{\partial F}{\partial \chi_{\bar{w}^{k} \bar{z}^{l}}^{2}} .
$$

Assume that $n_{1}>n_{2}+1$. Then differentiating $\tilde{D}_{w}(F)=0$ with respect to $u_{w \bar{w}^{k} \bar{z}^{l+1}}$ using (17) we find that $F$ is invariant with respect to the vector field

$$
Z=\frac{\partial}{\partial u_{\bar{w}^{k} \bar{z}^{l+1}}}+\frac{1}{u_{\bar{w}}^{2}} \frac{\partial}{\partial \chi_{\bar{w}^{k} \bar{z}^{l}}^{1}}
$$


Consequently, it is invariant with respect to the commutator

$$
\left[\tilde{D}_{w}, Z\right]=\tilde{D}_{w}\left(\frac{1}{u_{\bar{w}}^{2}}\right) \frac{\partial}{\partial \chi_{\bar{w}^{k} \bar{z}^{l}}^{1}}
$$

i.e., $F$ does not depend on $\chi_{\bar{w}^{k} \bar{z}^{l}}^{1}$ for $k+l=n_{1}$.

Set $n_{2}=n$; then $n_{1}=n+1$ and let $F \in \mathscr{F}(\mathscr{U}, n+1, n)$. The internal coordinates of maximal order on which the coefficients at $\partial / \partial \chi_{\bar{w}^{k} \bar{z}^{l}}^{1}$ and $\partial / \partial \chi_{\bar{w}^{k} \bar{z}^{l}}^{2}$ in $\tilde{D}_{w}(F)$ depend are

$$
\begin{aligned}
& u_{w \bar{w}^{n+1} \bar{z}}, u_{w \bar{w}^{n} \bar{z}^{2}}, \ldots, u_{w \bar{z}^{n+2}} \quad \text { for } \quad \frac{\partial}{\partial \chi_{\bar{w}^{k} \bar{z}^{l}}^{1}}, \\
& u_{w \bar{w}^{n+1} \bar{z}}, u_{w \bar{w}^{n} \bar{z}^{2}}, \ldots, u_{w \bar{w} \bar{z}^{n+1}} \quad \text { for } \quad \frac{\partial}{\partial \chi_{\bar{w}^{k} \bar{z}^{l}}^{2}} \text {. }
\end{aligned}
$$

Differentiating $\tilde{D}_{w}(F)=0$ with respect to these coordinates, we see that $F$ must be invariant with respect to the vector fields

$$
\begin{aligned}
Z_{0} & =\frac{\partial}{\partial u_{\bar{z}^{n+2}}}+\frac{1}{u_{\bar{w}}^{2}} \frac{\partial}{\partial \chi_{\bar{z}^{n+1}}^{1}}, \\
Z_{1} & =\frac{\partial}{\partial u_{\bar{w} \bar{z}^{n+1}}}+\frac{1}{u_{\bar{w}}^{2}} \frac{\partial}{\partial \chi_{\bar{w} \bar{z}^{n}}^{1}}+\frac{1}{2} \cdot\left(\frac{1}{u_{\bar{w}}^{2}}-\frac{1}{u_{\bar{w}}^{3}}\right) \chi^{1} \frac{\partial}{\partial \chi_{\bar{z}^{n}}^{2}} \\
& \cdots \\
Z_{i} & =\frac{\partial}{\partial u_{\bar{w}^{i} \bar{z}^{n-i+2}}}+\frac{1}{u_{\bar{w}}^{2}} \frac{\partial}{\partial \chi_{\bar{w}^{i} \bar{z}^{n-i+1}}^{1}}+\frac{1}{2} \cdot\left(\frac{1}{u_{\bar{w}}^{2}}-\frac{1}{u_{\bar{w}}^{3}}\right) \chi^{1} \frac{\partial}{\partial \chi_{\bar{w}^{i-1} \bar{z}^{n-i+1}}^{2}}, \\
& \ldots \\
Z_{n+1} & =\frac{\partial}{\partial u_{\bar{w}^{n+1} \bar{z}}}+\frac{1}{u_{\bar{w}}^{2}} \frac{\partial}{\partial \chi_{\bar{w}^{n+1}}^{1}}+\frac{1}{2} \cdot\left(\frac{1}{u_{\bar{w}}^{2}}-\frac{1}{u_{\bar{w}}^{3}}\right) \chi^{1} \frac{\partial}{\partial \chi_{\bar{w}^{n}}^{2}}
\end{aligned}
$$

Note that $\left[Z_{i}, Z_{j}\right]=0$.

We now use the induction on $n$. The base is $n=0$. Compute the commutator

$$
\left[\tilde{D}_{w}, Z_{0}\right]=-2 \frac{u_{w \bar{w}}}{u_{\bar{w}}^{2}} \frac{\partial}{\partial \chi_{\bar{z}}^{1}}-\frac{1}{u_{\bar{w}}^{2}} \cdot \frac{u_{w \bar{w}}}{u_{\bar{w}^{2}}} \frac{\partial}{\partial \chi^{2}} .
$$

Hence, $F$ is invariant with respect to

Next,

$$
Z_{0}^{1}=2 u_{\bar{w}} \frac{\partial}{\partial \chi_{\bar{z}}^{1}}+\frac{\partial}{\partial \chi^{2}}
$$

$$
\left[\tilde{D}_{w}, Z_{0}^{1}\right]=2 u_{w \bar{w}} \frac{\partial}{\partial \chi_{\bar{z}}^{1}}-2 u_{\bar{w}} \frac{u_{w \bar{w}}}{u_{\bar{w}}^{2}} \frac{\partial}{\partial \chi^{2}}=2 \frac{u_{w \bar{w}}}{u_{\bar{w}}}\left(u_{\bar{w}} \frac{\partial}{\partial \chi_{\bar{z}}^{1}}-\frac{\partial}{\partial \chi^{2}}\right)
$$

and thus the vector field

$$
Z_{0}^{2}=u_{\bar{w}} \frac{\partial}{\partial \chi_{\bar{z}}^{1}}-\frac{\partial}{\partial \chi^{2}}
$$

annihilates $F$ as well. But

$$
\frac{\partial}{\partial \chi^{2}}=\frac{1}{2}\left(Z_{0}^{1}-Z_{0}^{2}\right)
$$

which means that $F$ does not depend on $\chi^{2}$, i.e., we find ourselves in the situation of Step 1 .

Now pass to the induction step and assume $n>0$. Just as in the preceding computations, we see that the commutator $\left[\tilde{D}_{w}, Z_{0}\right]$ is proportional to the vector field

$$
Z_{0}^{1}=2 u_{\bar{w}} \frac{\partial}{\partial \chi_{\bar{z}^{n+1}}^{1}}+\frac{\partial}{\partial \chi_{\bar{z}^{n}}^{1}}
$$

while the commutator $\left[\tilde{D}_{w}, Z_{0}^{1}\right]$ is proportional to

$$
Z_{0}^{2}=u_{\bar{w}} \frac{\partial}{\partial \chi_{\bar{z}^{n+1}}^{1}}-\frac{\partial}{\partial \chi_{\bar{z}^{n}}^{2}}
$$


Thus, $F$ is independent of $\chi_{\bar{z}^{n}}^{2}$, so we change the field $Z_{1}$ to

$$
Z_{1}=\frac{\partial}{\partial u_{\bar{w} \bar{z}^{n+1}}}+\frac{1}{u_{\bar{w}}^{2}} \cdot \frac{\partial}{\partial \chi_{\bar{w}_{\bar{z}}^{n}}^{2}}
$$

Then $\left[\tilde{D}_{w}, Z_{1}\right]$ is proportional to the vector field

$$
Z_{1}^{1}=2 u_{\bar{w}} \frac{\partial}{\partial \chi_{\bar{w} \bar{z}^{n}}^{1}}+\frac{\partial}{\partial \chi_{\bar{w} \bar{z}^{n-1}}^{2}}
$$

while the commutator $\left[\tilde{D}_{w}, Z_{1}^{1}\right]$ equals, up to a factor, to the vector field

$$
Z_{1}^{2}=u_{\bar{w}} \frac{\partial}{\partial \chi_{\bar{w} \bar{z}^{n}}}-\frac{\partial}{\partial \chi_{\bar{w} \bar{z}^{n-1}}^{2}} .
$$

Hence, $F$ is independent of $\chi_{\bar{w}_{\bar{z}}^{n-1}}^{2}$ and instead of $Z_{2}$ we can take the vector field

$$
\frac{\partial}{\partial u_{\bar{w}^{2} \bar{z}^{n}}}+\frac{1}{u_{\bar{w}}^{2}} \frac{\partial}{\partial \chi_{\bar{w}^{2} \bar{z}^{n-1}}^{1}}
$$

etc. Eventually, we shall arrive at the independence of $F$ on all the variables $\chi_{\bar{w}^{i} \bar{z}^{n-1}}^{2}$, and this completes the induction step for $s=2$.

Step 3 (the induction step). Let $F \in \mathscr{F}\left(\mathscr{U}, n_{1}, \ldots, n_{s}\right)$

Lemma 3. One has $n_{j}=n_{j+1}+1, j=1, \ldots, s-1$, i.e., $n_{j}=n+s-j$, where $n=n_{s}$.

Proof of Lemma 3. The proof is similar to that of Lemma 2.

Thus, $F \in \mathscr{F}(\mathscr{U}, n+s-1, \ldots, n)$, and the internal coordinates of maximal jet order on which the coefficients in the total derivatives at $\partial / \partial \chi_{\bar{w}^{k} \bar{z}^{l}}^{i}$ that act nontrivially on $F$ may depend are

$$
\begin{aligned}
& u_{w \bar{w}^{n+s-1} \bar{z}}, u_{w \bar{w}^{n+s-2} \bar{z}^{2}}, \ldots, u_{w \bar{z}^{n+s}} \quad \text { for } \quad \frac{\partial}{\partial \chi_{\bar{w}^{k} \bar{z}^{l}}^{1}}, \\
& u_{w \bar{w}^{n+s-1} \bar{z}}, u_{w \bar{w}^{n+s-2} \bar{z}^{2}}, \ldots, u_{w \bar{w} \bar{z}^{n+s-1}} \quad \text { for } \quad \frac{\partial}{\partial \chi_{\bar{w}^{k} \bar{z}^{l}}^{2}} \text {. }
\end{aligned}
$$

Therefore, $F$ is invariant with respect to the vector fields

$$
\begin{aligned}
Z_{0} & =\frac{\partial}{\partial u_{\bar{z}^{n+s}}}+\frac{1}{u_{\bar{w}}^{2}} \frac{\partial}{\partial \chi_{\bar{z}^{n+s-1}}^{1}}, \\
Z_{1} & =\frac{\partial}{\partial u_{\bar{w} \bar{z}^{n+s-1}}}+\frac{1}{u_{\bar{w}}^{2}} \frac{\partial}{\partial \chi_{\bar{w} \bar{z}^{n+s-2}}^{1}}+\frac{1}{2} \cdot\left(\frac{1}{u_{\bar{w}}^{2}}-\frac{1}{u_{\bar{w}}^{3}}\right) \chi^{1} \frac{\partial}{\partial \chi_{\bar{z}^{n+s-2}}^{2}}, \\
& \cdots \\
Z_{i} & =\frac{\partial}{\partial u_{\bar{w}^{i} \bar{z}^{n+s-i}}}+\frac{1}{u_{\bar{w}}^{2}} \frac{\partial}{\partial \chi_{\bar{w}^{i} \bar{z}^{n+s-i-1}}^{1}}+\frac{1}{2} \cdot\left(\frac{1}{u_{\bar{w}}^{2}}-\frac{1}{u_{\bar{w}}^{3}}\right) \chi^{1} \frac{\partial}{\partial \chi_{\bar{w}^{i-1} \bar{z}^{n+s-i-1}}^{2}}, \\
& \ldots \\
Z_{n+s-1} & =\frac{\partial}{\partial u_{\bar{w}^{n+s-1} \bar{z}}}+\frac{1}{u_{\bar{w}}^{2}} \frac{\partial}{\partial \chi_{\bar{w}^{n+s-1}}^{1}}+\frac{1}{2} \cdot\left(\frac{1}{u_{\bar{w}}^{2}}-\frac{1}{u_{\bar{w}}^{3}}\right) \chi^{1} \frac{\partial}{\partial \chi_{\bar{w}^{n+s-2}}^{2}} .
\end{aligned}
$$

We first fix $s>2$ and set $n=0$ (the base of induction). Then

$$
Z_{0}=\frac{\partial}{\partial u_{\bar{z}}^{s}}+\frac{1}{u_{\bar{w}}^{2}} \cdot \frac{\partial}{\partial \chi_{\bar{z}^{s-1}}^{1}}
$$

Hence the commutator $\left[\tilde{D}_{w}, Z_{0}\right]$ is proportional to

$$
Z_{0}^{1}=2 u_{\bar{w}} \frac{\partial}{\partial \chi_{\bar{z}^{s-1}}^{1}}+\frac{\partial}{\partial \chi_{\bar{z}^{s-2}}^{2}},
$$

for the commutator $\left[\tilde{D}_{w}, Z_{0}^{1}\right]$ we get

$$
Z_{0}^{2}=3 u_{\bar{w}} \frac{\partial}{\partial \chi_{\bar{z}^{s-2}}^{2}}+\frac{\partial}{\partial \chi_{\bar{z}^{s-3}}^{3}},
$$


etc., and $\left[\tilde{D}_{w}, Z_{0}^{s-2}\right]$ equals, up to a functional factor, to the vector field

$$
Z_{0}^{s-1}=s u_{\bar{w}} \frac{\partial}{\partial \chi_{\bar{z}^{s-1}}}+\frac{\partial}{\partial \chi^{s}}
$$

Finally, for $\left[\tilde{D}_{w}, Z_{0}^{s-1}\right]$ we get

$$
Z_{0}^{s}=u_{\bar{w}} \frac{\partial}{\partial \chi_{\bar{z}}^{s-1}}-\frac{\partial}{\partial \chi^{s}}
$$

Therefore,

$$
\frac{\partial}{\partial \chi^{s}}=\frac{1}{s+1}\left(Z_{0}^{s-1}-s Z_{0}^{s}\right)
$$

and $F$ does not depend on $\chi^{s}$.

Let now $n>0$. Applying a similar procedure of step-by-step commutation with $Z_{0}$, we find that we have

$$
Z_{0}^{i}=\left[\tilde{D}_{w}, Z_{0}^{i-1}\right] \sim(i+1) u_{\bar{w}} \frac{\partial}{\partial \chi_{\bar{z}^{n+s-i}}^{i}}+\frac{\partial}{\partial \chi_{\bar{z}^{n+s-i-1}}^{i+1}}
$$

where $\sim$ denotes proportionality. In particular,

$$
Z_{0}^{s-1}=s u_{\bar{w}} \frac{\partial}{\partial \chi_{\bar{z}^{n+1}}^{s-1}}+\frac{\partial}{\partial \chi_{\bar{z}^{n}}^{s}}
$$

Then we have

$$
\left[\tilde{D}_{w}, Z_{0}^{s-1}\right] \sim u_{\bar{w}} \frac{\partial}{\partial \chi_{\bar{z}^{n+1}}^{s-1}}-\frac{\partial}{\partial \chi_{\bar{z}^{n}}^{s}}=Z_{0}^{s}
$$

This leads to the invariance of $F$ with respect to the fields $\partial / \partial \chi_{\bar{z}^{n+1}}^{s-1}$ and $\partial / \partial \chi_{\bar{z}^{n}}^{s}$. In addition, using equations (19)) we obtain by induction independence of $F$ on all the variables $\chi_{\bar{z}^{n+i}}^{s-i}$. In particular, this means that instead of $Z_{1}$ in equations (18) we can consider the vector field

$$
Z_{1}=\frac{\partial}{\partial u_{\bar{w} \bar{z}^{n+s-1}}}+\frac{1}{u_{\bar{w}}^{2}} \frac{\partial}{\partial \chi_{\bar{w} \bar{z}^{n+s-2}}^{1}} .
$$

Using (12) we get

$$
\left[\tilde{D}_{w}, Z_{1}\right]=-2 \frac{u_{w \bar{w}}}{u_{\bar{w}}^{3}} \frac{\partial}{\partial \chi_{\bar{w} \bar{z}^{n+s-2}}^{1}}-\frac{1}{u_{\bar{w}}^{2}}\left(\frac{u_{w \bar{w}}}{u_{\bar{w}}^{2}} \frac{\partial}{\partial \chi_{\bar{w} \bar{z}^{n+s-3}}^{2}}+\frac{u_{w \bar{z}}}{u_{\bar{w}}^{2}} \frac{\partial}{\partial \chi_{\bar{z}^{n+s-2}}^{2}}\right) .
$$

But, thanks to the previous remark, the last summand can be omitted and we can set

$$
Z_{1}^{1}=2 u_{\bar{w}} \frac{\partial}{\partial \chi_{\bar{w} \bar{z}^{n+s-2}}^{1}}+\frac{\partial}{\partial \chi_{\bar{w} \bar{z}^{n+s-3}}^{2}} .
$$

Proceeding in a similar fashion as above, we shall obtain the vector fields

$$
Z_{1}^{i}=(i+1) u_{\bar{w}} \frac{\partial}{\partial \chi_{\bar{w} \bar{z}^{n+s-i-1}}^{i}}+\frac{\partial}{\partial \chi_{\bar{w} \bar{z}^{n+s-i-2}}^{i+1}} .
$$

In particular,

$$
Z_{1}^{s-1}=s u_{\bar{w}} \frac{\partial}{\partial \chi_{\bar{w} \bar{z}^{n+s}}^{s-1}}+\frac{\partial}{\partial \chi_{\bar{w}^{n+s-1}}^{s}}
$$

and

$$
Z_{1}^{s}=u_{\bar{w}} \frac{\partial}{\partial \chi_{\bar{w}_{\bar{z}}^{n+s}}^{s-1}}-\frac{\partial}{\partial \chi_{\bar{w} \bar{z}^{n+s-1}}^{s}} .
$$

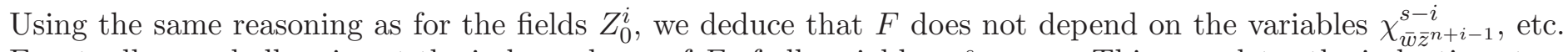
Eventually, we shall arrive at the independence of $F$ of all variables $\chi_{\bar{w}^{i} \bar{z}^{n+s-i}}^{s}$. This completes the induction step.

Theorem 1 is proved. 


\section{Closing Remarks}

In the present paper we have found infinitely many nonlocal conservation laws for the Przanowski equation and established their nontriviality.

To put these results into a context, recall that employing isospectral Lax pairs for the generation of conservation laws dates back to 1968, see [26] where an infinite hierarchy of local conservation laws for the celebrated Kortewegde Vries equation was constructed. However, as we have seen above, nonisospectral Lax pairs can be successfully employed for the same purpose too. A similar procedure was applied to other equations in [5, 17, 18, 32, and can be canonically associated to any differential covering in a geometrical framework, see [15]. On the other hand, while isospectral Lax pairs can also be applied for the construction of recursion operators in a fairly straightforward fashion, cf. e.g. [6, 11, 13, 27, 31] and references therein, to the best of our knowledge this does not seem to be the case for nonisospectral Lax pairs, especially in the case of more than two independent variables.

Note that the proof of nontriviality of the conservation laws obtained from a nonisospectral Lax pair can be quite hard, see the proof of Theorem 1 above, and, moreover, special handling of each particular equation could be required, cf. e.g. [5, 17, 18. The conservation laws resulting from the procedure in question are usually nonlocal, and it is natural to ask which is the use and meaning thereof.

Let $\mathscr{E}$ be a partial differential system in $m$ dependent variables $u^{A}$ and $n$ independent variables $x^{i}$, and $\tau: \tilde{\mathscr{E}} \rightarrow \mathscr{E}$ be a covering over this system, cf. e.g. [7, 20] for details. Then a $\tau$-nonlocal conservation law of $\mathscr{E}$ is nothing but a "usual", i.e., local, conservation law of the covering system $\tilde{\mathscr{E}}$. Thus, a $\tau$-nonlocal conservation law for $\mathscr{E}$ is a closed $(n-1)$-differential form on $\mathscr{E}$ whose coefficients can depend not just on the quantities $\frac{\partial^{|\sigma|} u^{A}}{\partial x^{\sigma}}$, but, informally speaking, also on the quantities like $\int \sum_{i=1}^{n} X_{i}^{\alpha} d x^{i}$, where

$$
\frac{\partial w^{\alpha}}{\partial x^{i}}=X_{i}^{\alpha}
$$

are the equations that define the nonlocal variables $w^{\alpha}$ in the covering $\tau$. So, in many respects, nonlocal conservation laws are quite similar to local ones, and share a number of possible applications with the latter.

For instance, for an evolutionary system nonlocal conservation laws yield constants of motion in essentially the same way as local ones. If, moreover, the evolutionary system under study admits a Hamiltonian structure, one can, under certain conditions, employ this structure to obtain (in general, nonlocal) symmetries from the nonlocal conserved densities in the same fashion as for the densities of local conservation laws, cf. e.g. 28]. On the qualitative level, the presence of an infinite hierarchy of nonlocal conservation laws, just as for that of their local counterparts, can be seen as indicative of integrability as it imposes strong constraints on the associated dynamics making the latter highly regular, cf. e.g. [1, 11] and references therein.

In closing note that our computations revealed only two local conservation laws of the Przanowski equation (2): $\omega_{1}$ given by formulas (44) and its "twin" arising as a result of the discrete symmetry mentioned at the end of Section 1, that is, $w \leftrightarrow \bar{w}, z \leftrightarrow \bar{z}$. Symbolic computations show that there exist no other local conservation laws of the order up to four. Unfortunately we have no rigorous nonexistence proof for arbitrarily high order, and in fact it would be quite interesting to find such a proof.

\section{ACKNowledgments}

The work of IK was partially supported by the RFBR Grant 18-29-10013 and IUM-Simons Foundation. The research of AS was supported in part by the Ministry of Education, Youth and Sport of the Czech Republic (MŠMT ČR) under RVO funding for IČ47813059 and the Grant Agency of the Czech Republic (GA ČR) under grant P201/12/G028. AS is pleased to thank A. Borowiec, M. Dunajski and K. Krasnov for stimulating discussions. AS also warmly thanks the Institute of Theoretical Physics of the University of Wrocław, and especially A. Borowiec, for the warm hospitality extended to him in the course of his visits to Wrocław, where some parts of the present article were worked on.

It is our great pleasure to thank the anonymous referee for useful and relevant comments.

\section{REFERENCES}

[1] M. Ablowitz, P.A. Clarkson, Solitons, nonlinear evolution equations and inverse scattering, Cambridge University Press, Cambridge, 1991.

[2] S. Alexandrov, B. Pioline, and S. Vandoren, Self-dual Einstein spaces, heavenly metrics, and twistors, J. Math. Phys. 51 (2010), 073510, arXiv:0912.3406

[3] M. Atiyah, M. Dunajski, L.J. Mason, Twistor theory at fifty: from contour integrals to twistor strings, Proc. R. Soc. A 473 (2017), no. 2206, art. 20170530 
[4] O. Babelon, D. Bernard, M. Talon, Introduction to classical integrable systems, Cambridge University Press, Cambridge, 2003.

[5] H. Baran, P. Blaschke, I.S. Krasil'shchik, M. Marvan, On symmetries of the Gibbons-Tsarev equation, J. Geom. Phys., to appear, https://doi.org/10.1016/j.geomphys.2019.05.011, arXiv:1811.08199

[6] H. Baran, I.S. Krasil'shchik, O.I. Morozov, P. Vojčák, Nonlocal Symmetries of Integrable Linearly Degenerate Equations: A Comparative Study, Theor. Math. Phys. 196 (2018), no. 2, 1089-1110, arXiv:1611.04938.

[7] A.V. Bocharov et al., Symmetries of Differential Equations in Mathematical Physics and Natural Sciences, edited by A.M. Vinogradov and I.S. Krasil'shchik). Factorial Publ. House, 1997 (in Russian). English translation: Amer. Math. Soc., 1999.

[8] P. Bull et al., Beyond $\Lambda$ CDM: Problems, solutions, and the road ahead, Physics of the Dark Universe 12 (2016), 56-99, arXiv:1512.05356

[9] S.P. Burtsev, V.E. Zakharov, A.V. Mikhailov, Inverse scattering method with variable spectral parameter, Theoret. and Math. Phys. 70 (1987), no. 3, 227-240.

[10] F. Calogero, Why are certain nonlinear PDEs both widely applicable and integrable?, in What is integrability?, ed. by V.E. Zakharov, Springer, Berlin, 1991, 1-62.

[11] M. Dunajski, Solitons, instantons, and twistors. Oxford University Press, Oxford, 2010.

[12] M. Hoegner, Quaternion-Kähler four-manifolds and Przanowski's function, J. Math. Phys. 53, 103517 (2012), arXiv:1205.3977

[13] B.G. Konopelchenko, Nonlinear integrable equations. Recursion operators, group-theoretical and Hamiltonian structures of soliton equations, Springer-Verlag, Berlin, 1987.

[14] V.E. Korepin, N.M. Bogoliubov, A.G. Izergin, Quantum inverse scattering method and correlation functions, Cambridge University Press, Cambridge, 1993.

[15] I.S. Krasil'shchik, A natural geometric construction underlying a class of Lax pairs, Lobachevskii J. of Math., 37 (2016) no. 1, 61-66, arXiv:1401.0612

[16] I.S. Krasil'shchik, Integrability in differential coverings, J. Geom. Phys. 87 (2015) 296-304, arXiv:1310.1189

[17] I.S. Krasil'shchik, A. Sergyeyev, Integrability of $S$-deformable surfaces: Conservation laws, Hamiltonian structures and more, J. Geom. Phys. 97 (2015), 266-278, arXiv:1501.07171

[18] I.S. Krasil'shchik, A. Sergyeyev, O.I. Morozov, Infinitely many nonlocal conservation laws for the $A B C$ equation with $A+B+C \neq 0$, Calc. Var. PDE 55 (2016), 1-12, arXiv:1511.09430

[19] I.S. Krasil'shchik and A.M. Vinogradov, Nonlocal trends in the geometry of differential equations: symmetries, conservation laws, and Bäcklund transformations, Acta Appl. Math. 15 (1989) 1-2, 161-209.

[20] J. Krasil'shchik, A. Verbovetsky, and R. Vitolo, The symbolic computation of integrability structures for partial differential equations, Springer, Texts \& Monographs in Symbolic Computation, 2017.

[21] K. Krasnov, Self-dual gravity, Class. Quantum Grav. 34 (2017) 095001, arXiv:1610.01457

[22] A. Lelito, O.I. Morozov, Three-component nonlocal conservation laws for Lax-integrable 3D partial differential equations. J. Geom. Phys. 131 (2018), 89-100.

[23] Z. Makridin, An effective algorithm for finding multidimensional conservation laws for integrable systems of hydrodynamic type, Theor. Math. Phys. 194 (2018), no. 2, 274-283.

[24] S.V. Manakov, P.M. Santini, Integrable dispersionless PDEs arising as commutation condition of pairs of vector fields, J. Phys. Conf. Ser. 482 (2014), 012029, arXiv:1312.2740

[25] L.J. Mason, N.M.J. Woodhouse, Integrability, self-duality, and twistor theory, Clarendon \& Oxford Univ. Press, N.Y., 1996.

[26] R.M. Miura, C.S. Gardner, and M.D. Kruskal, Korteweg-de Vries equation and generalizations. II. Existence of conservation laws and constants of motion. J. Math. Phys. 9 (1968) 1204-1209

[27] O.I. Morozov, A. Sergyeyev, The four-dimensional Martínez Alonso-Shabat equation: reductions and nonlocal symmetries, J. Geom. Phys. 85 (2014), 40-45, arXiv:1401.7942.

[28] P.J. Olver, Applications of Lie groups to differential equations, 2nd ed., Springer, N.Y., 1993.

[29] J.F. Plebański, Some solutions of complex Einstein equations, J. Math. Phys. 16 (1975), no. 12, 2395-2402.

[30] M. Przanowski, Locally Hermit-Einstein, self-dual gravitational instantons, Acta Phys. Polon. B14 (1983), 625-627.

[31] A. Sergyeyev, A simple construction of recursion operators for multidimensional dispersionless integrable systems, J. Math. Anal. Appl. 454 (2017), no. 2, 468-480, arXiv:1501.01955.

[32] A. Sergyeyev, New integrable (3+1)-dimensional systems and contact geometry, Lett. Math. Phys. 108 (2018), no. 2, 359-376, arXiv:1401.2122

[33] M.B. Sheftel, A.A. Malykh, Partner symmetries, group foliation and ASD Ricci-flat metrics without Killing vectors, SIGMA 9 (2013), 075, arXiv:1306.3195

[34] I.A.B. Strachan, The symmetry structure of the anti-self-dual Einstein hierarchy, J. Math. Phys. 36 (1995), 3566-3573, arXiv:hep-th/9410047

[35] E. Witten, Integrable lattice models from gauge theory, Adv. Theor. Math. Phys. 21 (2017), no. 7, 1819-1843, arXiv:1611.00592

[36] V.E. Zakharov, Integrable systems in multidimensional spaces, in Mathematical problems in theoretical physics (Berlin, 1981), Springer, Berlin, 1982, 190-216.

[37] V.E. Zakharov, Dispersionless limit of integrable systems in 2+1 dimensions, in Singular limits of dispersive waves (Lyon, 1991), Plenum, New York, 1994, 165-174.

V.A. Trapeznikov Institute of Control Sciences RaS, Profsoyuznaya 65, 117342 Moscow, Russia \& Independent University of Moscow, B. Vlasevsky 11, 119002 Moscow, Russia

E-mail address: josephkra@gmail.com

Mathematical Institute, Silesian University in Opava, Na RybníčKu 1, 74601 Opava, Czech Republic

E-mail address: artur.sergyeyev@math.slu.cz 